\section{OPEN ACCESS}

Edited by:

Abdallah Samy,

University of Kansas, United States

Reviewed by:

Ye Yuan,

Huazhong University of Science and

Technology, China

Taoyong Chen,

Second Military Medical

University, China

Yimin Li,

Guangzhou Medical University, China

*Correspondence:

Yiming Lu

bluesluyi@sina.com

Yan Shang

shangyan751200@163.com

Wenjun Chang

cwjcwj1976@smmu.edu.cn

Sheng Wang

wangsheng@tongji.edu.cn

tThese authors have contributed equally to this work

Specialty section:

This article was submitted to Infectious Diseases - Surveillance,

Prevention and Treatment,

a section of the journal

Frontiers in Medicine

Received: 28 April 2020

Accepted: 06 July 2020

Published: 17 July 2020

Citation:

Lu Y, Sun K, Guo S, Wang J, Li A,

Rong $X$, Wang $T$, Shang $Y$, Chang $W$ and Wang S (2020) Early Warning Indicators of Severe COVID-19: A Single-Center Study of Cases From Shanghai, China. Front. Med. 7:432. doi: 10.3389/fmed.2020.00432

\title{
Early Warning Indicators of Severe COVID-19: A Single-Center Study of Cases From Shanghai, China
}

\author{
Yiming Lu ${ }^{1,2,3 * t}$, Kuo Sun ${ }^{2 \dagger}$, Shanshan Guo ${ }^{3 \dagger}$, Junjie Wang ${ }^{1 \dagger}, \mathrm{An} \mathrm{Li}^{2}$, Xuli Rong ${ }^{3}$, \\ Tingfang Wang ${ }^{3}$, Yan Shang ${ }^{4 *}$, Wenjun Chang ${ }^{5 *}$ and Sheng Wang ${ }^{1 *}$
}

\begin{abstract}
'Department of Critical Care Medicine, School of Medicine, Shanghai Tenth People's Hospital, Tongji University, Shanghai, China, ${ }^{2}$ Department of Biochemical Pharmacy, School of Pharmacy, Second Military Medical University, Shanghai, China, ${ }^{3}$ School of Medicine, Shanghai University, Shanghai, China, ${ }^{4}$ Department of Respiratory and Critical Care Medicine, Changhai Hospital, Second Military Medical University, Shanghai, China, ${ }^{5}$ Department of Environment and Occupation Health, Second Military Medical University, Shanghai, China
\end{abstract}

Background: Patients with severe novel coronavirus disease (COVID-19) can likely develop comorbidities, which can lead to irreversible organ damage and, eventually, death. However, early indicators of disease progression remain unclear. This study aimed to identify early indicators of disease progression to provide a basis for improved prognostic prediction and disease management.

Methods: We examined 53 recovered adult COVID-19 patients who were treated at Shanghai Public Health Clinical Center between January 20, 2020, and February 20, 2020. The patients were categorized into the following four groups according to their condition at admission: mild condition $(n=3)$, moderate $(n=41)$, severe $(n=7)$, and critical $(n=2)$. They were also categorized according to disease progression as mild or moderate conditions that remained stable $(n=26)$, moderate disease that progressed to severe condition $(n=18)$, and continuously severe or critical $(n=9)$. We then focused on investigating the differences in the epidemiological and laboratory indicators between remained stable cases and progressed to severe condition cases.

Results: Mild or moderate patients were younger than severe or critical patients. The number of patients with shortness of breath and underlying diabetes and heart disease at admission was higher in the severe or critical group. This group also showed considerably lower or higher values in 28 laboratory indicators. In addition, mild and moderate patients who remained stable were younger than moderate patients progressing to severe disease. Men had a higher risk of disease progression. Patients who progressed had either higher or lower values in 11 laboratory indicators. Survival curve analysis showed that age, procalcitonin, D-dimer, serum C-reactive protein, lactate dehydrogenase, lymphocytes, neutrophils, CD4\%, and CD4/CD8 ratio were significant predictors of progression to severe disease.

Conclusions: Lactate dehydrogenase, procalcitonin, etc. are early warning indicators of severe COVID-19. Age (>64 years), shortness of breath, past histories of diabetes and 
heart disease, and abnormality in 28 other indicators at admission are indicative of severe or progression toward severe COVID-19. Meanwhile, abnormalities in 11 indicators and an abnormal coagulation function index at admission are risk factors for progression to severe disease.

Keywords: COVID-19, early warning indicators, severe patients, moderate patients, adult

\section{INTRODUCTION}

The novel coronavirus disease (COVID-19) has spread rapidly and become a pandemic. Several countries reported similar cases in 2019, but the specific origin of COVID-19 remained unidentified (1). Globally, by May 28, 2020, 5.79 million people were diagnosed with COVID-19, and the mortality reached 357,432 (2). Currently, COVID-19 is the most important health crisis worldwide. The latest COVID-19 research has focused on severely and critically ill patients because disease progression to this stage leads to rapid patient deterioration that can easily lead to inflammatory storm, respiratory distress, multiple organ dysfunction, and eventually, death. Therefore, the treatment of severe and critical cases is a priority. However, severe acute respiratory syndrome coronavirus 2 (SARS-CoV-2), which causes COVID-19, is highly transmissible; the available medical treatment modality is limited, with no standard curative modality identified to date. Thus, the treatment of severe COVID-19 has been extremely difficult $(3,4)$. Accordingly, it is important to explore early predictors of disease progression to provide timely intervention. In addition to categorizing the patient's condition as mild, moderate, severe, and critical, the "COVID-19 Treatment and Diagnosis Guidelines (Seventh Trial Version)" added three laboratory indicators namely, reduced lymphocyte count, inflammatory factors, and lactic acid level (5). A single-center retrospective study conducted in Wuhan Union Hospital reported that the reduction in lymphocytes and the pro-inflammatory cytokine storm are related to disease severity (6). With increasing research on severe COVID-19, T lymphocytes, lactic acid, and interleukin-6 (IL-6) have been identified to be helpful in the early identification of severe disease (7-10).

Although early indicators for severe COVID-19 have already been reported, the patients' general condition as well as the admission and treatment criteria differ across countries. Further, most of these criteria and evaluation systems are based on data from Wuhan, China, where the outbreak was first reported. Therefore, the values of these indicators or evaluation systems require further verification.

This study aimed to identify early indicators of disease progression to provide the basis for improved prognostic prediction and disease management. We included cases from Shanghai and selected indicators representative of mild, moderate, severe, and critical disease at admission, for analysis. In addition, this study focused on the comparison of general information, clinical manifestations, past histories, and laboratory indicators between moderate cases for which the disease progressed to severe condition and mild and moderate cases that remained stable.

\section{METHODS}

\section{Research Design and Subjects}

This study was approved by the Ethics Committee of Shanghai Public Health Clinical Center (SHPHC) (YJ-2020-S028-02). The need for informed consent was waived owing to the COVID-19 outbreak in 2019. Fifty-three COVID-19 patients who were admitted at and discharged after recovery from SHPHC between January 20, 2020, and February 20, 2020, were included. Diagnoses were confirmed according to the "COVID-19 and Diagnosis Guidelines of the National Health Commission of the People's Republic of China (Fifth Trial Edition)" and reviewed according to the "COVID-19 and Diagnosis Guideline of the National Health Commission of the People's Republic of China (Seventh Trial Edition)." All the patients were examined for consistent items immediately after admission. The patients were managed with the same program (the same laboratory tests and the same general principles of treatment were applied). Although the approach to management differs according to the individual situation of each patient, the management itself did not vary when compared with the clinical and laboratory manifestations. Patients whose data were mostly missing were excluded. A patient was categorized as having mild severity if the following criteria were met: mild disease was defined as mild clinical symptoms, but no pneumonia symptoms observed on imaging. Moderate disease was defined as the occurrence of fever and respiratory symptoms as well as pneumonia features observed on imaging. Severe disease was defined if one of the following criteria was met: (1) shortness of breath, with respiratory rate (RR) $\geq 30$ beats/min; (2) blood oxygen saturation $\leq 93 \%$ under the resting state; (3) partial pressure of oxygen $\left(\mathrm{PaO}_{2}\right) /$ fraction of inspired oxygen $\left(\mathrm{FiO}_{2}\right) \leq 300 \mathrm{mmHg}$; and (4) significant disease progression $>50 \%$ in lung images within $24-$ $48 \mathrm{~h}$. Finally, a patient was identified as having critical disease if, in addition to the above criteria, one of the following was met: (1) respiratory failure that requires mechanical ventilation; (2) shock; and (3) multiple organ failure that requires intensive care unit (ICU) monitoring.

\section{Data Collection}

Data at admission, laboratory test results, and imaging examination results were collected from the electronic medical records and nursing records. The data were validated through direct inspection by the medical officers in charge, thereby ensuring data integrity. At admission, we collected data on age, sex, contact history, chronic disease history (hypertension, diabetes, malignancy, heart disease, lung disease, liver disease, kidney disease, and thyroid disease), and admission symptoms (fever, cough, sputum, chest tightness, shortness of breath, 
headache, myalgia, diarrhea, nausea, poor appetite, inappetence, and fatigue).

\section{Statistical Analysis}

Data on age and time from onset to admission were reported as median (interquartile range), whereas other continuous variables were expressed as mean \pm standard deviation (SD). Comparisons were conducted using the independent sample $t$-test, Fisher's exact test, and $\chi^{2}$-test. The primary study outcome was the identification of indicators with the highest early predictive value based on the survival curves. The survival curves were plotted from the optimal statistical time windows of mild and moderate cases that remained stable and moderate cases that progressed to severe conditions in MaxStat software. The secondary outcome was the identification of supportive indicators for disease progression after admission. We combined mild and moderate patients into one group and severe and critically ill patients into another group and analyzed the various laboratory tests between the two groups to explore the early warning indicators of COVID-19. Pairwise comparisons were subsequently conducted between data from mild and moderate cases and from severe and critical cases. Then, we used the same group (mild and moderate patients) mentioned above and analyzed the various laboratory tests between the patients of this group who progressed to severe and stable conditions. In addition, trends in continuous data of patients with mild and moderate conditions that remained stable and those with mild and moderate disease that progressed to severe conditions were analyzed. For all analyses, $P<0.05$ was considered statistical significance.

\section{RESULTS}

\section{Patient Characteristics}

Of the 53 patients, 26 had moderate disease at admission, and their conditions were stable during the treatment; 18 had moderate disease at admission, and the disease progressed to severe during the treatment; 9 had severe or critical disease at admission, and the condition did not improve. The patients were categorized into four groups according to their condition at admission: mild $(n=3)$, moderate $(n=41)$, severe $(n=7)$, and critical $(n=2)$. They were also categorized according to disease progression into those with mild or moderate conditions that remained stable $(n=26)$, those with moderate disease that progressed to severe conditions $(n=18)$, and those whose conditions were continuously severe or critical $(n=9)$. The patient characteristics are shown in Table 1. The median patients' age in the overall cohort was 60.5 (range, 41.3-67.5) years. Mild or moderately ill patients were significantly younger than severely or critically ill patients (median age: 54 [range, 40.5-64.5] vs. 70 [range, 60.0-75.0] years, $p=0.001)$. The majority of the patients were male ( 34 vs. 19). The most common symptom was fever (83.02\%), followed by cough $(37.74 \%)$ and sputum production (24.53\%). In addition, compared with the mildly or moderately ill group, there were more patients in the severely and critically ill group who experienced shortness of breath $(p=0.005)$. However, there were no significant differences in other symptoms such as fever, cough, sputum, chest tightness, fatigue, and gastrointestinal
TABLE 1 | Baseline information of COVID-19 patients enrolled in this study.

\begin{tabular}{ccc}
\hline All patients & $\begin{array}{c}\text { Mild and } \\
\text { moderate } \\
(n=44)\end{array}$ & $\begin{array}{c}\text { Severe and } P \text {-value* } \\
\text { critical } \\
(n=9)\end{array}$
\end{tabular}

Age, Median (IQR)-yrs 60.5 (41.3-67.5) $54(40.5-64.5) 70$ (60.0-75.0) $0.0010^{\dagger}$

Gender, $n$ (\%)

\begin{tabular}{|c|c|c|c|c|}
\hline & \\
\hline Male & $34(64.15)$ & 26 (59.09) & 8 (88.89) & \\
\hline Female & $19(35.85)$ & $18(40.91)$ & $1(11.11)$ & \\
\hline Exposure, $n(\%)$ & & & & 0.50 \\
\hline Wuhan-direct & 26 (49.06) & $21(47.73)$ & $5(55.56)$ & \\
\hline Wuhan-indirect & $13(24.53)$ & $10(22.73)$ & $3(33.33)$ & \\
\hline No explicit contact & $14(26.42)$ & $13(29.55)$ & $1(11.11)$ & \\
\hline $\begin{array}{l}\text { Onset to admission, } \\
\text { Median (IQR)-days }\end{array}$ & $4(3-7)$ & $4(3-7)$ & $4(3-10)$ & 0.71 \\
\hline \multicolumn{5}{|l|}{ Main symptoms, $n(\%)$} \\
\hline No any symptoms & $3(5.66)$ & $3(6.82)$ & $0(0.00)$ & 0.42 \\
\hline Fever & 44 (83.02) & 37 (84.09) & $7(77.78)$ & 0.65 \\
\hline Cough & $20(37.74)$ & $18(40.91)$ & $2(22.22)$ & 0.46 \\
\hline Headache & $3(5.66)$ & $3(6.82)$ & $0(0.00)$ & 0.42 \\
\hline Myalgia & $2(3.77)$ & $2(4.55)$ & $0(0.00)$ & 0.43 \\
\hline Sputum production & $13(24.53)$ & $12(27.27)$ & $1(11.11)$ & 0.31 \\
\hline Diarrhea & $1(1.89)$ & $1(2.27)$ & $0(0.00)$ & 0.65 \\
\hline Chest tightness & $7(13.21)$ & $5(11.36)$ & $2(22.22)$ & 0.38 \\
\hline Anhelation & $6(11.32)$ & $2(4.55)$ & $4(44.44)$ & 0.0050 \\
\hline Dyspnea & $1(1.89)$ & $0(0.00)$ & $1(11.11)$ & 0.17 \\
\hline Fatigue & $9(16.98)$ & $7(15.91)$ & $2(22.22)$ & 0.64 \\
\hline Nausea & $2(3.77)$ & $2(4.55)$ & $0(0.00)$ & 0.51 \\
\hline Poor appetite & $5(9.43)$ & $4(9.09)$ & $1(11.11)$ & 0.85 \\
\hline \multicolumn{5}{|l|}{ Underlying disease } \\
\hline Hypertension & $16(30.19)$ & $12(27.27)$ & $4(44.44)$ & 0.43 \\
\hline Diabetes & $5(9.43)$ & $2(4.55)$ & 3 (33.33) & 0.030 \\
\hline Liver disease & $2(3.77)$ & $1(2.27)$ & $1(11.11)$ & 0.31 \\
\hline Lung disease & $3(5.66)$ & $2(4.55)$ & $1(11.11)$ & 0.44 \\
\hline Heat disease & $7(13.21)$ & $3(6.82)$ & $4(44.44)$ & 0.012 \\
\hline Thyroid disease & $2(3.77)$ & $2(4.55)$ & $0(0.00)$ & 0.54 \\
\hline Kidney disease & $1(1.89)$ & $0(0.00)$ & $1(11.11)$ & 0.17 \\
\hline Cancers & $2(3.77)$ & $1(2.27)$ & $1(11.11)$ & 0.31 \\
\hline
\end{tabular}

Data are median (IQR), $n(\%)$, or $n / N(\%)$, where $N$ is the total number of patients with available data. P-values compare patients with mild \& moderate disease with patients with severe \& critical disease using $\chi^{2}$-test, Fisher's exact test, or student $t$-test. * ${ }^{2}{ }^{2}$-test or Fisher's exact test, ${ }^{\dagger}$ Student $t$-test.

discomfort between the two groups. Over $70 \%$ of the patients had underlying diseases including 5 (9.43\%) with diabetes, 16 (30.19\%) with hypertension, 7 (13.21\%) with heart disease, and $2(3.77 \%)$ with cancer. The proportions of patients with diabetes $(p=0.030)$ and heart disease $(p=0.012)$ were significantly higher in the severely and critically ill group than in the mild and moderately ill group.

The severely and critically ill group showed significantly higher results in 16 laboratory indicators than the mild and moderately ill group including B-type natriuretic peptide precursor $(p<0.0001)$, C-reactive protein (CRP) $(p=0.0040)$, D-2 polymer $(p=0.0080)$, IgA $(p=0.0030)$, leukocyte count 
TABLE 2 | Comparison of laboratory examination between patients with mild \& moderate or severe \& critical types disease.

\begin{tabular}{|c|c|c|c|}
\hline & $\begin{array}{c}\text { Mild and } \\
\text { moderate } \\
(N=44)\end{array}$ & $\begin{array}{l}\text { Severe and } \\
\text { critical } \\
(N=9)\end{array}$ & \\
\hline & Mean \pm SD & Mean \pm SD & $P$-value* \\
\hline \multicolumn{4}{|l|}{ Blood routine } \\
\hline Leukocyte count, $\times 10^{9}$ per $\mathrm{L}$ & $4.64 \pm 1.74$ & $9.21 \pm 6.23$ & $<0.0001$ \\
\hline Lymphocyte count, $\times 10^{9}$ per $\mathrm{L}$ & $1.09 \pm 0.74$ & $0.58 \pm 0.29$ & 0.049 \\
\hline Lymphocyte, \% & $22.28 \pm 8.90$ & $9.47 \pm 8.08$ & $<0.0001$ \\
\hline Neutrophil count, $\times 10^{9}$ per $\mathrm{L}$ & $3.10 \pm 1.36$ & $8.09 \pm 6.09$ & $<0.0001$ \\
\hline Neutrophil, \% & $67.59 \pm 10.04$ & $83.69 \pm 11.23$ & $<0.0001$ \\
\hline Monocyte count, $\times 10^{9}$ per $\mathrm{L}$ & $2.69 \pm 11.41$ & $0.49 \pm 0.38$ & 0.57 \\
\hline Monocyte, \% & $9.25 \pm 4.77$ & $6.21 \pm 4.33$ & 0.084 \\
\hline Eosinophil count, $\times 10^{9}$ per $\mathrm{L}$ & $0.02 \pm 0.07$ & $0.03 \pm 0.08$ & 0.70 \\
\hline Eosinophil, \% & $0.23 \pm 0.51$ & $0.47 \pm 1.10$ & 0.31 \\
\hline \multicolumn{4}{|l|}{ Blood biochemistry } \\
\hline $\begin{array}{l}\text { Partial blood oxygen pressure, } \\
\mathrm{KPa}\end{array}$ & $13.45 \pm 4.86$ & $14.32 \pm 7.07$ & 0.65 \\
\hline Hemoglobin, g/L & $15.84 \pm 3.37$ & $15.12 \pm 2.27$ & 0.54 \\
\hline Standard bicarbonate, $\mathrm{mmol} / \mathrm{L}$ & $24.26 \pm 1.61$ & $22.59 \pm 2.90$ & 0.019 \\
\hline Residual alkali, mmol/L & $-0.34 \pm 2.28$ & $-2.49 \pm 3.60$ & 0.026 \\
\hline Oxygen saturation, \% & $97.24 \pm 1.92$ & $95.94 \pm 3.08$ & 0.11 \\
\hline Carbon dioxide, $\mathrm{mmol} / \mathrm{L}$ & $21.01 \pm 2.33$ & $20.08 \pm 2.11$ & 0.27 \\
\hline Lactate, mmol// & $2.53 \pm 0.78$ & $3.46 \pm 1.07$ & 0.0030 \\
\hline Bilirubin (Arterial blood), $\mu \mathrm{mol} / \mathrm{L}$ & $9.47 \pm 5.88$ & $15.44 \pm 9.90$ & 0.018 \\
\hline L- $\gamma$-glutamyltransferase, $\mathrm{U} / \mathrm{L}$ & $46.60 \pm 42.25$ & $39.00 \pm 36.34$ & 0.62 \\
\hline Glutathione reductase, U/L & $79.54 \pm 17.63$ & $101.90 \pm 25.67$ & 0.0020 \\
\hline Alkaline phosphatase, U/L & $55.82 \pm 22.01$ & $57.44 \pm 9.99$ & 0.83 \\
\hline Lactate dehydrogenase, U/L & $284.70 \pm 109.62$ & $425.33 \pm 132.62$ & 0.0010 \\
\hline Total bilirubin, $\mu \mathrm{mol} / \mathrm{L}$ & $9.32 \pm 4.53$ & $11.03 \pm 5.28$ & 0.32 \\
\hline Direct bilirubin, $\mu \mathrm{mol} / \mathrm{L}$ & $6.12 \pm 10.85$ & $6.34 \pm 3.33$ & 0.95 \\
\hline Total protein, g/L & $68.43 \pm 5.32$ & $64.08 \pm 6.22$ & 0.035 \\
\hline Albumin, $g / l$ & $38.91 \pm 5.40$ & $33.31 \pm 5.62$ & 0.0070 \\
\hline A $/$ G, \% & $1.63 \pm 1.55$ & $1.10 \pm 0.26$ & 0.32 \\
\hline Prealbumin, $g / L$ & $119.51 \pm 45.66$ & $77.44 \pm 45.89$ & 0.015 \\
\hline Apolipoprotein A, g/L & $0.95 \pm 0.19$ & $0.78 \pm 0.12$ & 0.0090 \\
\hline Low density lipoprotein, $\mathrm{mmol} / \mathrm{L}$ & $2.80 \pm 1.13$ & $3.58 \pm 1.30$ & 0.072 \\
\hline High density lipoprotein, mmol/L & $1.22 \pm 0.48$ & $0.89 \pm 0.21$ & 0.052 \\
\hline Glucose, mmol/L & $7.07 \pm 1.55$ & $13.61 \pm 8.93$ & $<0.001$ \\
\hline Uric acid, $\mu \mathrm{mol} / \mathrm{L}$ & $257.00 \pm 98.52$ & $330.02 \pm 215.73$ & 0.12 \\
\hline Haptoglobin, mg/dl & $234.61 \pm 99.92$ & $280.61 \pm 96.84$ & 0.21 \\
\hline Acid glycoprotein, mg/dl & $170.38 \pm 44.73$ & $187.69 \pm 37.37$ & 0.28 \\
\hline $\begin{array}{l}\text { B-type natriuretic peptide } \\
\text { precursor, pg/ml }\end{array}$ & $106.25 \pm 213.62$ & $679.95 \pm 653.75$ & $<0.0001$ \\
\hline Creatinine, $\mu \mathrm{mol} / \mathrm{L}$ & $70.18 \pm 18.24$ & $120.98 \pm 111.87$ & 0.006 \\
\hline Retinol binding protein, mg/L & $22.53 \pm 7.47$ & $20.78 \pm 9.77$ & 0.55 \\
\hline Total calcium, mmol/L & $2.01 \pm 0.10$ & $1.90 \pm 0.12$ & 0.0090 \\
\hline \multicolumn{4}{|l|}{ Coagulation function } \\
\hline D-dimer, $\mu \mathrm{g} / \mathrm{ml}$ & $1.01 \pm 2.98$ & $4.89 \pm 6.65$ & 0.0080 \\
\hline INR & $1.04 \pm 0.18$ & $1.06 \pm 0.11$ & 0.67 \\
\hline PTA, \% & $102.70 \pm 19.55$ & $93.11 \pm 14.08$ & 0.17 \\
\hline $\begin{array}{l}\text { Activated partial thromboplastin } \\
\text { time, } s\end{array}$ & $41.98 \pm 9.26$ & $40.08 \pm 6.37$ & 0.56 \\
\hline
\end{tabular}

(Continued)
TABLE 2 | Continued

\begin{tabular}{|c|c|c|c|}
\hline & $\begin{array}{c}\text { Mild and } \\
\text { moderate } \\
(N=44)\end{array}$ & $\begin{array}{l}\text { Severe and } \\
\text { critical } \\
(N=9)\end{array}$ & \\
\hline & Mean \pm SD & Mean \pm SD & $P$-value* \\
\hline Thrombin time, s & $16.28 \pm 1.10$ & $18.13 \pm 2.32$ & 0.0010 \\
\hline Prothrombin time, s & $13.42 \pm 0.95$ & $13.94 \pm 1.15$ & 0.15 \\
\hline Fibrinogen, g/l & $4.61 \pm 1.18$ & $5.69 \pm 1.75$ & 0.027 \\
\hline \multicolumn{4}{|l|}{ Immunology } \\
\hline $\mathrm{C} 3, \mathrm{~g} / \mathrm{l}$ & $1.13 \pm 0.25$ & $1.13 \pm 0.27$ & 0.99 \\
\hline CD3+ lymphocyte, \% & $65.02 \pm 13.88$ & $48.20 \pm 6.68$ & 0.0010 \\
\hline CD3+ lymphocyte count, $/ \mu \mathrm{L}$ & $720.82 \pm 691.59$ & $274.00 \pm 183.26$ & 0.062 \\
\hline CD4+ lymphocyte, \% & $38.73 \pm 11.11$ & $27.72 \pm 8.30$ & 0.0070 \\
\hline CD4+ lymphocyte count, $/ \mu \mathrm{L}$ & $384.48 \pm 173.96$ & $163.11 \pm 147.02$ & 0.0010 \\
\hline CD8+ lymphocyte, \% & $23.35 \pm 11.10$ & $18.63 \pm 6.78$ & 0.23 \\
\hline CD8+ lymphocyte count, $/ \mu \mathrm{L}$ & $303.59 \pm 598.73$ & $102.78 \pm 67.25$ & 0.32 \\
\hline CD45+ lymphocyte count, $/ \mu \mathrm{L}$ & $1029.84 \pm 753.77$ & $545.67 \pm 286.88$ & 0.065 \\
\hline CD4/CD8 & $1.94 \pm 0.82$ & $1.76 \pm 1.08$ & 0.56 \\
\hline $\lg A, g / l$ & $2.29 \pm 0.93$ & $3.57 \pm 1.77$ & 0.0030 \\
\hline $\lg G, g / l$ & $12.06 \pm 1.79$ & $12.22 \pm 2.66$ & 0.83 \\
\hline $\operatorname{lgM}, g / l$ & $1.06 \pm 0.48$ & $1.05 \pm 0.44$ & 0.96 \\
\hline \multicolumn{4}{|l|}{ Infection-related biomarkers } \\
\hline C-reactive protein, mg/l & $38.01 \pm 41.03$ & $94.60 \pm 84.99$ & 0.0040 \\
\hline $\begin{array}{l}\text { Erythrocyte sedimentation rate, } \\
\mathrm{mm} / \mathrm{h}\end{array}$ & $64.77 \pm 35.76$ & $80.13 \pm 28.77$ & 0.26 \\
\hline Procalcitonin, ng/ml & $0.14 \pm 0.33$ & $0.54 \pm 0.79$ & 0.015 \\
\hline
\end{tabular}

Laboratory indicators of mild \& moderate or severe \& critical patients with available data. $P$ values compare patients with mild \& moderate disease with patients with severe \& critical

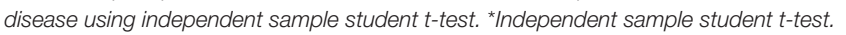

$(p<0.0001)$, procalcitonin $(p=0.015)$, thrombin time $(p=$ $0.0010)$, lactate $(p=0.0030)$, lactate dehydrogenase $(p=0.0010)$, total calcium $(p=0.0090)$, and neutrophil count $(p<0.0001)$ (Table 2). In contrast, the severely and critically ill group showed significantly lower values than the mild and moderately ill group in 12 laboratory indicators including CD3 percentage $(p=$ $0.0010)$, absolute CD4 count $(p=0.0070)$, albumin $(p=0.0070)$, and lymphocyte count $(p=0.049)$ (Table 2).

Older age patients, those with underlying disease including diabetes and heart disease, male patients, and those with significantly higher or lower values in one of the above laboratory indicators often experienced disease progression and had poor prognosis. This indicates that these factors may predict the progression to severe disease.

\section{Comparison Between Mild and Moderate Patients That Progressed to Severe Disease or Remained Stable}

For further analysis of the early warning indicators of disease progression, we compared the prognosis among the 44 patients with mild or moderate disease at admission. Of these, 26 remained stable and were eventually cured and discharged. Further, 18 patients developed progression to 
TABLE 3 | Baseline information of COVID-19 mild and moderate patients with or without progressive disease.

\begin{tabular}{|c|c|c|c|}
\hline & $\begin{array}{l}\text { Stable disease } \\
\quad(n=26)\end{array}$ & $\begin{array}{l}\text { Progressive disease } \\
\qquad(n=18)\end{array}$ & $P$-value* \\
\hline Age, Median (IQR)-yrs & $48.0(40.0-59.0)$ & $64.0(49.5-69.5)$ & $0.045^{\dagger}$ \\
\hline \multicolumn{4}{|l|}{ Gender, $n(\%)$} \\
\hline Male & $12(46.15)$ & $14(77.78)$ & 0.036 \\
\hline Female & $14(53.85)$ & $4(22.22)$ & \\
\hline Exposure, $n(\%)$ & & & 0.61 \\
\hline Wuhan-direct & $14(53.85)$ & 7 (38.89) & \\
\hline Wuhan-indirect & $5(19.23)$ & $5(27.78)$ & \\
\hline No explicit contact & 7 (26.92) & $6(33.33)$ & \\
\hline $\begin{array}{l}\text { Onset to admission, Median } \\
\text { (IQR)-days }\end{array}$ & $4.0(3.0-6.5)$ & $5.0(3.0-6.8)$ & 0.53 \\
\hline \multicolumn{4}{|l|}{ Main symptoms, $n$ (\%) } \\
\hline No any symptoms & $3(11.54)$ & $0(0.00)$ & 0.26 \\
\hline Fever & $20(76.92)$ & $17(94.44)$ & 0.21 \\
\hline Cough & $11(42.31)$ & 7 (38.89) & 0.82 \\
\hline Headache & $3(11.54)$ & $0(0.00)$ & 0.26 \\
\hline Myalgia & $2(7.69)$ & $0(0.00)$ & 0.23 \\
\hline Sputum production & 7 (26.92) & $5(27.78)$ & 0.95 \\
\hline Diarrhea & $1(3.85)$ & $0(0.00)$ & 0.40 \\
\hline Chest tightness & $3(11.54)$ & $2(11.11)$ & 0.97 \\
\hline Anhelation & $1(3.85)$ & $1(5.56)$ & 0.79 \\
\hline Fatigue & $6(23.08)$ & $1(5.56)$ & 0.21 \\
\hline Nausea & $2(7.69)$ & $0(0.00)$ & 0.23 \\
\hline Poor appetite & $3(11.54)$ & $1(5.56)$ & 0.63 \\
\hline \multicolumn{4}{|l|}{ Underlying disease, $n$ (\%) } \\
\hline Hypertension & $5(19.23)$ & 7 (38.89) & 0.18 \\
\hline Diabetes & $0(0.00)$ & $2(11.11)$ & 0.16 \\
\hline Liver disease & $0(0.00)$ & $1(5.56)$ & 0.41 \\
\hline Lung disease & $0(0.00)$ & $2(11.11)$ & 0.16 \\
\hline Heat disease & $1(3.85)$ & $2(11.11)$ & 0.35 \\
\hline Thyroid disease & $1(3.85)$ & $1(5.56)$ & 0.79 \\
\hline Cancers & $1(3.85)$ & $0(0.00)$ & 0.40 \\
\hline
\end{tabular}

Data are median (IQR), $n(\%)$, or $n / N(\%)$, where $N$ is the total number of patients with available data. P-values compare mild \& moderate patients with stable disease with patients with progressive disease using $\chi^{2}$-test, Fisher's exact test, or student $t$-test. ${ }^{*} \chi^{2}$-test or Fisher's exact test, ${ }^{\dagger}$ Student $t$-test.

severe disease. Analysis of the demographic characteristics, past histories, admission symptoms, and laboratory test results of these two groups showed that those with disease progression were significantly older (mean age, 64.0 [range, 49.5-69.5] vs. 49.0 [40.0-59.0] years, $p=0.045$ ) and were higher in males (77.78 vs. $46.15 \%, p=0.036)$ (Table 3). The remaining clinical manifestations (e.g., representative symptoms such as fever, cough, fatigue, and myalgia) and underlying diseases (e.g., diabetes, hypertension, and heart disease) were not significantly different between the two groups (Table 3).

Compared with the stable group, the disease progression group had significantly lower CD3\% $(p=0.032)$, CD4\% ( $p=$ $0.0060)$, absolute CD4 lymphocyte count $(p=0.0040)$, and total calcium level $(p=0.0020)$ as well as significantly higher CRP ( $p$ $=0.0010)$ and lactate dehydrogenase $(p=0.0010)$ values, along with higher values of 5 other indicators (Table 4). Factors such as people of an old age (older), man, and significantly higher or lower value in one of the above laboratory indicators were associated with disease progression. The patients in the disease progression group also had blood coagulation as well as liver and kidney function disorders. These results indicate that these factors can be used as early indicators for disease progression in COVID-19 patients.

\section{Indicators for Disease Progression}

With hospital admission as the starting point, and disease progression to severe condition as the end point, the longest duration to disease progression was 12 days, whereas the longest duration to stable condition was 15 days. To investigate the correlation between indicators and disease progression in COVID-19, survival curves were plotted in the optimal statistical time windows (maximally selected rank statistics with several $p$-value approximations) (11) of mild and moderate cases whose conditions remained stable, and moderate cases that progressed to severe conditions using MaxStat software. In total, 9 significant early indicators were identified including age (>64 years), procalcitonin, D-dimer, serum CRP, lactate dehydrogenase, lymphocytes, neutrophils, CD4\%, and CD4/CD8 ratio (Figure 1). These factors were indicative of disease initiation and progression to severe COVID-19 during the early stage of the disease.

\section{DISCUSSION}

Currently, the incidence and mortality rates of COVID-19 have exceeded those of severe acute respiratory syndrome (SARS) in 2003 (8,273 confirmed cases and 775 deaths) and the Middle East respiratory syndrome (1,139 confirmed cases and 431 deaths). COVID-19 is also more easily transmissible than these two illnesses $(12,13)$. Although most COVID19 patients only exhibit mild and moderate symptoms, some patients develop severe disease, which can lead to death. Patient management is further complicated by challenges in the treatment of severe COVID-19 cases due to complicated patient conditions, restricted treatment environment, and the absence of a specific curative strategy. Therefore, early indicators of disease initiation and progression to severe condition would be crucial for reducing the morality rate and improving prognosis.

Research on COVID-19 has shown that elderly male patients with comorbidities are at the highest risk of infection (8). Similarly, we found that people of an old age and man predict progression in patients. In addition, while elderly COVID-19 patients with underlying illness such as cerebrovascular disease, liver disease, kidney disease, or malignancy often die owing to their original comorbidities $(3,14)$, we found that the presence of underlying disease itself was a risk factor for progression to severe COVID-19. While a previous study showed that patients with hypertension have a higher risk of COVID-19 (10), we found no evidence to indicate that hypertension can be an early indicator of 
TABLE 4 | Comparison of Laboratory examination between mild and moderate patients with or without progressive disease.

\begin{tabular}{|c|c|c|c|}
\hline & $\begin{array}{l}\text { Stable disease } \\
\quad(n=26)\end{array}$ & $\begin{array}{c}\text { Progressive } \\
\text { disease }(n=18)\end{array}$ & \\
\hline & Mean \pm SD & Mean \pm SD & $P$-value* \\
\hline \multicolumn{4}{|l|}{ Blood routine } \\
\hline Leukocyte count, $\times 10^{9}$ per $\mathrm{L}$ & $4.29 \pm 1.33$ & $5.16 \pm 2.14$ & 0.10 \\
\hline Lymphocyte count, $\times 10^{9}$ per $\mathrm{L}$ & $1.09 \pm 0.30$ & $1.08 \pm 1.13$ & 0.95 \\
\hline Lymphocyte, \% & $24.03 \pm 7.42$ & $19.74 \pm 10.38$ & 0.12 \\
\hline Neutrophil count, $\times 10^{9}$ per $\mathrm{L}$ & $2.75 \pm 1.08$ & $3.60 \pm 1.58$ & 0.040 \\
\hline Neutrophil, \% & $65.19 \pm 7.23$ & $71.06 \pm 12.52$ & 0.056 \\
\hline Monocyte count, $\times 10^{9}$ per $\mathrm{L}$ & $4.24 \pm 14.75$ & $0.46 \pm 0.43$ & 0.29 \\
\hline Monocyte, \% & $9.68 \pm 3.73$ & $8.62 \pm 6.04$ & 0.47 \\
\hline Eosinophil count, $\times 10^{9}$ per $\mathrm{L}$ & $0.03 \pm 0.08$ & $0.01 \pm 0.06$ & 0.50 \\
\hline Eosinophil, \% & $0.28 \pm 0.42$ & $0.14 \pm 0.61$ & 0.37 \\
\hline \multicolumn{4}{|l|}{ Blood biochemistry } \\
\hline $\begin{array}{l}\text { Partial blood oxygen pressure, } \\
\mathrm{KPa}\end{array}$ & $13.27 \pm 4.54$ & $13.72 \pm 5.44$ & 0.77 \\
\hline Hemoglobin, g/L & $15.28 \pm 2.75$ & $16.70 \pm 4.07$ & 0.18 \\
\hline Standard bicarbonate, $\mathrm{mmol} / \mathrm{L}$ & $24.54 \pm 1.30$ & $23.82 \pm 1.96$ & 0.15 \\
\hline Residual alkali, mmol/L & $0.15 \pm 1.76$ & $-1.05 \pm 2.76$ & 0.096 \\
\hline Oxygen saturation, \% & $97.45 \pm 1.78$ & $96.92 \pm 2.13$ & 0.38 \\
\hline Carbon dioxide, mmol/L & $21.55 \pm 1.66$ & $20.19 \pm 2.96$ & 0.060 \\
\hline Lactate, mmol/l & $2.49 \pm 0.89$ & $2.58 \pm 0.58$ & 0.71 \\
\hline Bilirubin (Arterial blood), $\mu \mathrm{mol} / \mathrm{L}$ & $8.44 \pm 5.68$ & $11.06 \pm 5.99$ & 0.16 \\
\hline$L$ - $\gamma$-glutamyltransferase, $\mathrm{U} / \mathrm{L}$ & $44.15 \pm 45.12$ & $50.35 \pm 38.46$ & 0.64 \\
\hline Glutathione reductase, $U / L$ & $75.18 \pm 14.57$ & $86.21 \pm 20.15$ & 0.043 \\
\hline Alkaline phosphatase, U/L & $56.00 \pm 26.43$ & $55.53 \pm 13.42$ & 0.95 \\
\hline Lactate dehydrogenase, U/L & $240.42 \pm 87.77$ & $352.41 \pm 107.00$ & 0.0010 \\
\hline Total bilirubin, $\mu \mathrm{mol} / \mathrm{L}$ & $8.22 \pm 3.24$ & $11.01 \pm 5.70$ & 0.047 \\
\hline Direct bilirubin, $\mu \mathrm{mol} / \mathrm{L}$ & $6.67 \pm 13.95$ & $5.28 \pm 1.89$ & 0.69 \\
\hline Total protein, g/L & $69.68 \pm 4.67$ & $66.50 \pm 5.80$ & 0.054 \\
\hline Albumin, g/l & $40.20 \pm 3.02$ & $36.93 \pm 7.45$ & 0.051 \\
\hline $\mathrm{A} / \mathrm{G}, \%$ & $1.78 \pm 2.01$ & $1.40 \pm 0.14$ & 0.44 \\
\hline Prealbumin, g/L & $129.84 \pm 45.69$ & $103.72 \pm 42.09$ & 0.066 \\
\hline Apolipoprotein A, g/L & $0.99 \pm 0.20$ & $0.89 \pm 0.15$ & 0.069 \\
\hline Low density lipoprotein, mmol/L & $3.04 \pm 1.28$ & $2.45 \pm 0.76$ & 0.097 \\
\hline High density lipoprotein, mmol/L & $1.29 \pm 0.59$ & $1.11 \pm 0.21$ & 0.23 \\
\hline Glucose, mmol/L & $7.03 \pm 1.69$ & $7.14 \pm 1.36$ & 0.83 \\
\hline Uric acid, $\mu \mathrm{mol} / \mathrm{L}$ & $244.02 \pm 63.12$ & $276.85 \pm 136.25$ & 0.29 \\
\hline Haptoglobin, mg/dl & $235.78 \pm 89.23$ & $232.81 \pm 117.31$ & 0.93 \\
\hline Acid glycoprotein, mg/dl & $160.65 \pm 45.59$ & $185.27 \pm 40.17$ & 0.077 \\
\hline $\begin{array}{l}\text { B-type natriuretic peptide } \\
\text { precursor, pg/ml }\end{array}$ & $63.04 \pm 68.42$ & $172.34 \pm 323.73$ & 0.10 \\
\hline Creatinine, $\mu \mathrm{mol} / \mathrm{L}$ & $63.44 \pm 14.05$ & $80.48 \pm 19.44$ & 0.0020 \\
\hline Retinol binding protein, mg/L & $23.53 \pm 7.47$ & $21.01 \pm 7.44$ & 0.29 \\
\hline Total calcium, mmol/L & $2.04 \pm 0.09$ & $1.95 \pm 0.10$ & 0.0020 \\
\hline \multicolumn{4}{|l|}{ Coagulation function } \\
\hline D-dimer, $\mu \mathrm{g} / \mathrm{ml}$ & $0.52 \pm 0.29$ & $1.76 \pm 4.72$ & 0.19 \\
\hline INR & $1.03 \pm 0.20$ & $1.04 \pm 0.13$ & 0.89 \\
\hline PTA, \% & $106.38 \pm 21.45$ & $97.06 \pm 15.12$ & 0.13 \\
\hline $\begin{array}{l}\text { Activated partial thromboplastin } \\
\text { time, s }\end{array}$ & $39.68 \pm 4.65$ & $45.51 \pm 13.02$ & 0.042 \\
\hline
\end{tabular}

(Continued)
TABLE $4 \mid$ Continued

\begin{tabular}{|c|c|c|c|}
\hline & $\begin{array}{l}\text { Stable disease } \\
\qquad(n=26)\end{array}$ & $\begin{array}{c}\text { Progressive } \\
\text { disease }(n=18)\end{array}$ & \\
\hline & Mean \pm SD & Mean \pm SD & $P$-value* \\
\hline Thrombin time, s & $16.15 \pm 0.81$ & $16.47 \pm 1.44$ & 0.35 \\
\hline Prothrombin time, s & $13.23 \pm 0.54$ & $13.72 \pm 1.32$ & 0.10 \\
\hline Fibrinogen, g/l & $4.76 \pm 1.20$ & $4.38 \pm 1.15$ & 0.31 \\
\hline \multicolumn{4}{|l|}{ Immunology } \\
\hline C3, g/l & $1.17 \pm 0.22$ & $1.07 \pm 0.27$ & 0.19 \\
\hline CD3+ lymphocyte, \% & $68.72 \pm 9.82$ & $59.68 \pm 17.16$ & 0.032 \\
\hline CD3+ lymphocyte count, $/ \mu \mathrm{L}$ & $720.85 \pm 213.42$ & $720.78 \pm 1069.03$ & 1.0 \\
\hline CD4+ lymphocyte, \% & $42.42 \pm 8.98$ & $33.39 \pm 11.95$ & 0.0060 \\
\hline CD4+ lymphocyte count, $/ \mu \mathrm{L}$ & $445.50 \pm 157.75$ & $296.33 \pm 161.34$ & 0.0040 \\
\hline CD8+ lymphocyte, \% & $22.70 \pm 6.84$ & $24.29 \pm 15.52$ & 0.65 \\
\hline CD8+ lymphocyte count, $/ \mu \mathrm{L}$ & $238.85 \pm 93.88$ & $397.11 \pm 937.07$ & 0.40 \\
\hline CD4/CD8 & $2.04 \pm 0.70$ & $1.81 \pm 0.97$ & 0.37 \\
\hline CD45+ lymphocyte count, $/ \mu \mathrm{L}$ & $1025.35 \pm 310.78$ & $1036.33 \pm 1137.98$ & 0.96 \\
\hline $\lg A, g / l$ & $2.40 \pm 1.02$ & $2.12 \pm 0.78$ & 0.35 \\
\hline $\lg G, g / l$ & $12.29 \pm 1.77$ & $11.72 \pm 1.82$ & 0.31 \\
\hline $\operatorname{lgM}, \mathrm{g} / \mathrm{l}$ & $1.10 \pm 0.47$ & $1.00 \pm 0.50$ & 0.50 \\
\hline \multicolumn{4}{|l|}{ Infection-related biomarkers } \\
\hline C-reactive protein, mg/l & $21.64 \pm 25.28$ & $62.57 \pm 48.21$ & 0.0010 \\
\hline $\begin{array}{l}\text { Erythrocyte sedimentation rate, } \\
\mathrm{mm} / \mathrm{h}\end{array}$ & $68.38 \pm 38.08$ & $59.56 \pm 32.46$ & 0.43 \\
\hline Procalcitonin, ng/ml & $0.07 \pm 0.13$ & $0.26 \pm 0.48$ & 0.063 \\
\hline
\end{tabular}

Laboratory indicators of mild or moderate conditions that remained stable patients or moderate disease that progressed to severe condition patients with available data. $P$-values compare mild \& moderate patients with stable disease with patients with progressive disease using independent sample student $t$-test. ${ }^{*} / n d e p e n d e n t ~ s a m p l e$ student $t$-test.

COVID-19. This finding may be owing to our small sample size, and the relationship between hypertension and COVID-19 needs to be further explored. Although hypertension was the most common underlying disease in the included patients, there was no significant difference in the rate of hypertension according to prognosis. Further, patients with diabetes and heart disease were more likely to become severely ill, which could possibly be due to an immunocompromised state and metabolic dysfunction.

The clinical manifestations of COVID-19 are viral pneumonia symptoms including fever, fatigue, dry cough, and diarrhea. Fever is the most common clinical manifestation of COVID19 , followed by cough $(15,16)$. In addition, chest tightness and shortness of breath are also important symptoms. In this study, the proportion of severe and critical cases with shortness of breath at admission was significantly higher than that of mild and moderate cases. Furthermore, analysis of the symptoms of all severe and critical cases in this study suggested that shortness of breath was often accompanied by accelerated disease progression in lung images. Several laboratory indicators have also been proposed as early indicators of severe COVID-19 (17, 18). For example, it has been reported that IL-6 and lactic acid can independently predict the progression of COVID-19 (10). Further, increased CRP and progressive 

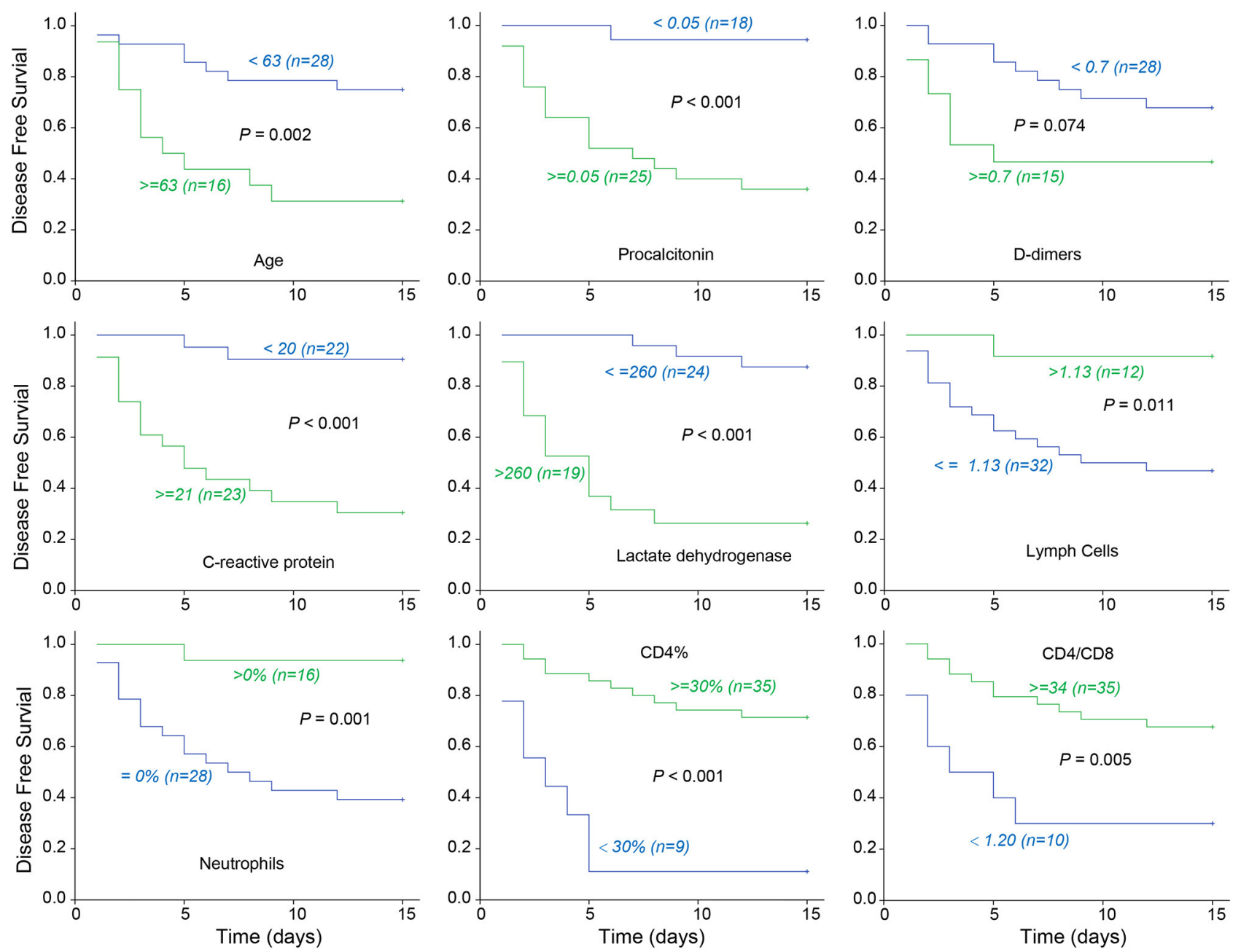

FIGURE 1 | COVID-19 early warning indicator survival curve. The associations between severe disease free survival and early indicators in 43 patients with mild or moderate disease upon admission. Taking hospitalization to become severe as the end, the latest turn weight is 12 days, and the longest stable condition is 15 days. The optimal cut-points are selected for discriminating the patients to high or low risk who will develop severe disease using Maxstat software. Lactate dehydrogenase and procalcitonin etc. are early warning indicators to severe COVID-19.

decrease in the absolute lymphocyte count have also been observed in severe COVID-19 patients (19-21). Therefore, these factors have been incorporated in the "COVID-19 Diagnosis and Treatment Guidelines (Seventh Trial Version)" issued by the National Health Commission of the People's Republic of China as early indicators of severe COVID-19 cases. In line with these findings, our results showed that increased CRP and decline of absolute lymphocyte count occurred in severe COVID-19 patients. We also found a significant difference in lactic acid index between the severely ill group and the mild or moderately ill group. This result indicates that lactic acid could be an early indicator of disease progression. In addition, we found that lactate dehydrogenase showed significant difference in both comparisons. This was later verified in the survival curve analysis. An elevated lactate dehydrogenase level may reduce the effectiveness of lactic acid as an early indicator of COVID19. As elevated lactate dehydrogenase activity indicates early myocardial injury, it has been adopted as an indicator for acute myocardial infarction (22). High lactate dehydrogenase levels are also associated with tissue injury occurring in various diseases, including pulmonary disorders such as pneumonia, and liver and kidney dysfunctions; therefore, corresponding treatments should be taken timeously to prevent further deterioration of the disease (23-25). Similarly, as COVID-19 can also cause pneumonia as 
well as heart, liver, kidney, and other organ dysfunctions, the patients may die from heart failure, shock, acute respiratory distress syndrome, arrhythmia, or renal failure (16). Therefore, lactate dehydrogenase plays a vital role as an early indicator of disease initiation and progression to severe disease. Yan et al. also confirmed lactate dehydrogenase and other indicators as crucial predictive biomarkers of disease mortality (24).

Studies have reported that the severity of pulmonary infection and immune injury in all patients with SARS 2003 was associated with the infiltration of large numbers of neutrophils and macrophages in the lungs. Similar events were observed in patients with COVID-19 $(26,27)$. After the onset of COVID-19, CD4+T lymphocytes are immediately activated and become pathogenic type $1 \mathrm{~T}$ helper (Th1) cells that produce granulocyte-macrophage colony-stimulating factor, and accelerate the inflammatory response (28). In our study, we found that the absolute count and percentage of $\mathrm{CD} 4+\mathrm{T}$ lymphocytes were significantly lower in the disease progression group than in the stable group. In addition, survival curve analysis indicated that the reduction of $\mathrm{CD} 4 / \mathrm{CD} 8$ ratio was closely related to the development of disease. Procalcitonin, a calcitonin-related gene product expressed in human epithelial cells in response to bacterial infections, was substantially increased in this study. Procalcitonin levels increase within 6$12 \mathrm{~h}$ of infection in response to pro-inflammatory mediator release after bacterial invasion and correlate with disease severity and clinical outcome, in patients with infection (29); it can also indicate the occurrence of sepsis (30). Procalcitonin is more specific for bacterial infections than CRP or white cell count $(29,31)$. Its elevation indicates the appearance of secondary infection in COVID-19 patients, which is an important warning signal, indicating that patients have low immune function and that the disease has reached the progressive stage. Significant decreases or increases in the above indicators are possibly associated with immune function disorders during disease progression.

We also found that an abnormal coagulation function indicator was associated with disease initiation and progression in severe COVID-19 cases. Aside from a substantially higher D-dimer level, other indicators, including the thrombin time, prothrombin time, and fibrinogen content were also higher in these cases than in the mild and moderate cases. Concurrently, the survival curve analysis indicated that the $\mathrm{D}$-dimer level was higher in the disease progression group than in the stable group. Overall, these findings indicate that severely ill COVID-19 patients developed blood coagulation function disorders and were at a certain stage of coagulation or bleeding. Other studies reported similar conclusions that COVID-19 patients in the ICU demonstrate higher prothrombin time and D-dimer level at admission, and their median D-dimer level is higher than that of non-ICU patients $(14,20)$. In addition, a recent autopsy of a patient who died of COVID19 revealed extensive bleeding and abnormal thrombosis in the lung tissue (32). These results indicate that coagulation indicators can predict disease initiation and progression in severe COVID-19 cases.
This study has some limitations. First, although we included a total of 177 confirmed cases from January 20 to February 20, 2020, the COVID-19 outbreak was declared a Public Health Emergency of International Concern, with incomplete detection indicators in some cases while some patients were still at the hospitalization stage and remained under intensive care. Therefore, we included only 53 patients with relatively complete and representative data in the analysis, including all basic information and laboratory indicators of all types of patients, including all 27 patients with severe and critical disease. Second, this was a retrospective study. Although our data can facilitate early diagnosis and prognostic prediction of severe COVID-19, the findings need to be further verified.

In conclusion, age and laboratory indicators, such as lactate dehydrogenase, procalcitonin, and D-dimer, are early predictors of severe COVID-19. Shortness of breath at admission, past histories of diabetes and heart disease, and abnormalities in the 28 indicators, such as CD4 percentage and CRP, indicate that the patient is already severely ill or has a significant risk of progressing to severe conditions. Meanwhile, abnormalities in 11 indicators, such as CD4 percentage after admission, are risk factors for progression to severe condition. Moreover, coagulation function disorder is also an early indicator of the disease.

\section{DATA AVAILABILITY STATEMENT}

The raw data supporting the conclusions of this article will be made available by the authors, without undue reservation.

\section{ETHICS STATEMENT}

This study was approved by the Ethics Committee of SHPHC (YJ2020-S028-02). The need for informed consent was waived due to the COVID-19 outbreak in 2019.

\section{AUTHOR CONTRIBUTIONS}

YL, SW, WC, YS, and JW designed the study, take responsibility for the integrity of the data, and the accuracy of data analysis. YL, SW, WC, and KS were responsible for conducting the study protocol and collecting all pertinent data. KS, SG, JW, AL, and XR performed the statistical analysis and drafted the manuscript. SW, WC, and TW revised the final manuscript. All authors contributed to the article and approved the submitted version.

\section{FUNDING}

This study was funded by the Emergency Project of Shanghai Science and Technology Commission (20411950200). The study sponsor was involved in the decision to submit the paper for publication, but was not involved in the study design and patient recruitment, the collection, analysis and interpretation of the data, and the writing of this manuscript. None of the authors were paid to draft this article by a pharmaceutical 
company or any other agencies. As the corresponding authors, we had full access to all the data in this study and had final responsibility for the decision to submit the manuscript for publication.

\section{REFERENCES}

1. Remuzzi A, Remuzzi G. COVID-19 and Italy: what next? Lancet. (2020) 395:1225-8. doi: 10.1016/S0140-6736(20)30627-9

2. COVID-19 Coronavirus Pandemic. Available online at: https://www. worldometers.info/coronavirus/ (accessed May 28, 2020).

3. Wang D, Hu B, Hu C, Zhu F, Liu X, Zhang J, et al. Clinical characteristics of 138 hospitalized patients with 2019 novel coronavirus-infected pneumonia in Wuhan, China. JAMA. (2020). doi: 10.1001/jama.2020.1585

4. Xu X, Yu C, Qu J, Zhang L, Jiang S, Huang D, et al. Imaging and clinical features of patients with 2019 novel coronavirus SARS-CoV-2. Eur J Nucl Med Mol Imaging. (2020) 47:1275-80. doi: 10.1007/s00259-020-04735-9

5. The Notice of Launching Guideline on Diagnosis and Treatment of the Novel Coronavirus Pneumonia (NCP). 7th ed. National Health Commission of the People's Republic of China. Available online at: http://www.nhc.gov.cn/yzygj/ s7653p/202003/46c9294a7dfe4cef80dc7f5912eb1989.shtml (accessed March 22, 2020).

6. Liu J, Li S, Liu J, Liang B, Wang X, Wang H, et al. Longitudinal characteristics of lymphocyte responses and cytokine profiles in the peripheral blood of SARS-CoV-2 infected patients. EBioMedicine. (2020) 55:102763. doi: 10.1016/j.ebiom.2020.102763

7. Vincent JL, de Mendonça A, Cantraine F, Moreno R, Takala J, Suter $\mathrm{PM}$, et al. Use of the SOFA score to assess the incidence of organ dysfunction/failure in intensive care units: results of a multicenter, prospective study. Working group on "sepsis-related problems" of the European Society of Intensive Care Medicine. Crit Care Med. (1998) 26:1793800. doi: 10.1097/00003246-199811000-00016

8. Chen N, Zhou M, Dong X, Qu J, Gong F, Han Y, et al. Epidemiological and clinical characteristics of 99 cases of 2019 novel coronavirus pneumonia in Wuhan, China: a descriptive study. Lancet. (2020) 395:507-13. doi: 10.1016/S0140-6736(20)30211-7

9. Diao B, Wang C, Tan Y, Chen X, Liu Y, Ning L, et al. Reduction and functional exhaustion of T cells in patients with coronavirus disease 2019 (COVID-19). Front Immunol. (2020) 11:827. doi: 10.1101/2020.02.18.20024364

10. Yang P, Ding Y, Xu Z, Pu R, Li P, Yan J, et al. Epidemiological and clinical features of COVID-19 patients with and without pneumonia in Beijing, China. MedRxiv. (2020). doi: 10.1101/2020.02.28.20028068

11. Lausen B, Schumacher M. Maximally selected rank statistics. Biometrics. (1992) 48:73-85. doi: 10.2307/2532740

12. Peeri NC, Shrestha N, Rahman MS, Zaki R, Tan Z, Bibi S, et al. The SARS, MERS and novel coronavirus (COVID-19) epidemics, the newest and biggest global health threats: what lessons have we learned? Int J Epidemiol. (2020) dyaa033. doi: 10.1093/ije/dyaa033. [Epub ahead of print].

13. Wang Y, Wang Y, Chen Y, Qin Q. Unique epidemiological and clinical features of the emerging 2019 novel coronavirus pneumonia (COVID19) implicate special control measures. J Med Virol. (2020) 92:568-76. doi: 10.1002/jmv.25748

14. Huang C, Wang Y, Li X, Ren L, Zhao J, Hu Y, et al. Clinical features of patients infected with 2019 novel coronavirus in Wuhan, China. Lancet. (2020) 395:497-506. doi: 10.1016/S0140-6736(20)30183-5

15. Lai CC, Shih TP, Ko WC, Tang HJ, Hsueh PR. Severe acute respiratory syndrome coronavirus 2 (SARS-CoV-2) and coronavirus disease-2019 (COVID-19): the epidemic and the challenges. Int J Antimicrob Agents. (2020) 55:105924. doi: 10.1016/j.ijantimicag.2020.105924

16. Wang T, Du Z, Zhu F, Cao Z, An Y, Gao Y, et al. Comorbidities and multi-organ injuries in the treatment of COVID-19. Lancet. (2020) 395:e52. doi: 10.1016/S0140-6736(20)30558-4

17. Li M, Dong Y, Wang H, Guo W, Zhou H, Zhang Z, et al. Cardiovascular disease potentially contributes to the progression and poor prognosis of COVID-19. Nutr Metab Cardiovasc Dis. (2020) 7:1061-7. doi: 10.1016/j.numecd.2020.04.013

\section{ACKNOWLEDGMENTS}

We thank all the patients for participating in this study. We thank Editage (www.editage.cn) for English language editing.

18. Lipsitch M, Swerdlow DL, Finelli L. Defining the epidemiology of Covid-19 - studies needed. N Engl J Med. (2020) 382:1194-6. doi: 10.1056/NEJMp2002125

19. Aggarwal S, Garcia Telles N, Aggarwal G, Lavie C, Lippi G, Henry BM. Clinical features, laboratory characteristics, and outcomes of patients hospitalized with coronavirus disease 2019 (COVID-19): early report from the United States. Diagnosis. (2020) 7:91-6. doi: 10.1515/dx-2020-0046

20. Lake MA. What we know so far: COVID-19 current clinical knowledge and research. Clin Med. (2020) 20:124-7. doi: 10.7861/clinmed.2019-coron

21. Singhal T. A review of coronavirus disease-2019 (COVID-19). Indian J Pediatr. (2020) 87:281-6. doi: 10.1007/s12098-020-03263-6

22. Gudbjarnason S, Priver DM. LDH-isoenzymes in infarcted heart muscle. Life Sci. (1968) 7:623-7. doi: 10.1016/0024-3205(68)90084-2

23. Kishaba T, Tamaki H, Shimaoka Y, Fukuyama H, Yamashiro S. Staging of acute exacerbation in patients with idiopathic pulmonary fibrosis. Lung. (2014) 192:141-9. doi: 10.1007/s00408-013-9530-0

24. Yan L, Zhang HT, Goncalves J, Xiao Y, Wang M, Guo Y, et al. An interpretable mortality prediction model for COVID-19 patients. Nat Mach Intell. (2020) 2:283-8. doi: 10.1038/s42256-020-0180-7

25. Zheng Z, Peng F, Xu B, Zhao J, Liu H, Peng J, et al. Risk factors of critical and mortal COVID-19 cases: a systematic literature review and meta-analysis. $J$ Infect. (2020). doi: 10.1016/j.jinf.2020.04.021. [Epub ahead of print].

26. Nicholls JM, Poon LLM, Lee KC, Ng WF, Lai ST, Leung CY, et al. Lung pathology of fatal severe acute respiratory syndrome. Lancet. (2003) 361:17738. doi: 10.1016/S0140-6736(03)13413-7

27. Gu J, Gong E, Zhang B, Zheng J, Gao Z, Zhong Y, et al. Multiple organ infection and the pathogenesis of SARS. J Exp Med. (2005) 202:415-24. doi: 10.1084/jem.20050828

28. Zhou Y, Fu B, Zheng X, Wang D, Zhao C, Qi Y, et al. Berrant pathogenic GM-CSF+ T cells and inflammatory CD14+CD16+ monocytes in severe pulmonary syndrome patients of a new coronavirus. bioRxiv. (2020). doi: $10.1101 / 2020.02 .12 .945576$

29. Schuetz P, Wirz Y, Sager R, Christ-Crain M, Stolz D, Tamm $\mathrm{M}$, et al. Effect of procalcitonin-guided antibiotic treatment on mortality in acute respiratory infections: a patient level meta-analysis. Lancet Infect Dis. (2018) 18:95-107. doi: 10.1016/S1473-3099(18) 30016-1

30. Christ Crain M, Müller B. Procalcitonin in bacterial infections-hype, hope, more or less? Swiss Med Week. (2005) 135:451-60.

31. Rowland T, Hilliard H, Barlow G. Procalcitonin: potential role in diagnosis and management of sepsis. Adv Clin Chem. (2015) 68:71-86. doi: 10.1016/bs.acc.2014.11.005

32. Xu Z, Shi L, Wang Y, Zhang J, Huang L, Zhang C, et al. Pathological findings of COVID-19 associated with acute respiratory distress syndrome. Lancet Respir Med. (2020) 8:420-2. doi: 10.1016/S2213-2600(20) 30076-X

Conflict of Interest: The authors declare that the research was conducted in the absence of any commercial or financial relationships that could be construed as a potential conflict of interest.

The reviewer TC declared an affiliation with no collaboration, with several of the authors, YL, KS, YS, and WC, to the handling editor at the time of review.

Copyright (C) 2020 Lu, Sun, Guo, Wang, Li, Rong, Wang, Shang, Chang and Wang. This is an open-access article distributed under the terms of the Creative Commons Attribution License (CC BY). The use, distribution or reproduction in other forums is permitted, provided the original author(s) and the copyright owner(s) are credited and that the original publication in this journal is cited, in accordance with accepted academic practice. No use, distribution or reproduction is permitted which does not comply with these terms. 